\title{
Contribution to the Control of Fire-induced Smoke Flow in Longitudinally Ventilated Tunnels
}

\author{
STEPHANE GAILLOT ${ }^{1}$, ALISTAIR REVELL ${ }^{1}$, DOMINIQUE BLAY ${ }^{1}$, \\ JEAN-PIERRE VANTELON ${ }^{2}$, and PASCAL DEBERTEIX ${ }^{3}$ \\ ${ }^{1}$ Laboratoire d'Etudes Thermiques, UMR CNRS 6608 \\ Ecole Nationale Supérieure de Mécanique et d'Aérotechnique \\ University of Poitiers \\ 1 avenue Clément Ader \\ BP 40109 -86961 Futuroscope Chasseneuil, France \\ ${ }^{2}$ Laboratoire de Combustion et de Détonique, UPR CNRS 9028 \\ Ecole Nationale Supérieure de Mécanique et d'Aérotechnique \\ University of Poitiers \\ 1 avenue Clément Ader \\ BP 40109 - 86961 Futuroscope Chasseneuil, France \\ ${ }^{3}$ RATP \\ Contrôle Général de Sécurité/Sécurité Incendie \\ 105 Boulevard de Chanzy \\ 93100 Montreuil, France
}

\begin{abstract}
This work looks at the longitudinal ventilation of fires in a tunnel and the associated phenomenon of backlayer formation. Computational fluid Dynamics are used to simulate the flow and to give predictions that can be compared with experimental data. Focusing in upon the use of Richardson number, different reference temperatures are investigated and it is found that using a temperature calculated directly from the heat release rate of the fire will yield the most useful results. Experimental results are compared to the numerical prediction showing a reasonable level of agreement. The use of a volumetric heat source to model the fire is also validated.

That accuracy of the results depends heavily on heat transfer to the wall and roof, is evidenced.

Finally, the basic requirement of any scale experiment being that its results are transferable to real-world case it is trying to represent, the existence of a Super Critical Ventilation Velocity of $\approx 3 \mathrm{~ms}^{-1}$, independent of the power of the fire, above which all the smokes produced are pushed downstream, is verified.
\end{abstract}

KEYWORDS: tunnel fire, longitudinal ventilation, smoke flow, backlayering

\section{NOMENCLATURE}

\begin{tabular}{|c|c|c|c|}
\hline A & Section of tunnel $\left(\mathrm{m}^{2}\right)$ & $T_{\text {ref }}$ & Reference temperature (K) \\
\hline$C_{p}$ & Specific heat $\left(\mathrm{Jkg}^{-1} \mathrm{~K}^{-1}\right)$ & $T_{o}$ & Ambient temperature $(\mathrm{K})$ \\
\hline$D_{H}$ & Hydraulic diameter (m) & $u$ & $\begin{array}{l}\text { Longitudinal ventilation velocity } \\
\left(\mathrm{ms}^{-1}\right)\end{array}$ \\
\hline$g$ & Acceleration of gravity $\left(\mathrm{ms}^{-2}\right)$ & $v$ & $\begin{array}{l}\text { Velocity component in y direction } \\
\left(\mathrm{ms}^{-1}\right)\end{array}$ \\
\hline$H$ & Height of tunnel (m) & $x$ & Longitudinal coordinate (m) \\
\hline l & Characteristic reference length (m) & $y$ & Vertical coordinate (m) \\
\hline
\end{tabular}




$\begin{array}{ll}L & \text { Backlayer length (m) } \\ P f & \text { Power of fire (W) } \\ R i & \text { Richardson number } \\ T & \text { Temperature (K) }\end{array}$

\section{INTRODUCTION}

Only over the last two decades has much attention been paid to the danger of tunnel fires. Before this, the design of a tunnel ventilation system was based on the fresh air required to maintain acceptable air quality.

Fortunately, tunnel fires are a rarity. But their character moreoften dramatic (in a recent past over two hundred lives have been lost in tunnel fires in Europe alone (Mont Blanc, Kaprun, St Gothard,...)) lead to review these events in order to help prevent future accidents. Safety measures that may now appear obvious may well have once been recommended as a result of an accident. Now, the designers are forced to carefully consider the tunnel fire scenario, because fire and smoke control are increasingly emerging as limiting factors.

Most ventilation systems use longitudinal ventilation, i.e., air flowing coaxial with the traffic direction. It is the smoke and heat, not the fire itself, that are truly deadly and so an effective ventilation system can prevent disaster. If the ventilation is of insufficient magnitude to force all the fumes to flow in one direction only, the flow is split into two regions: upstream and downstream of the fire. There is a layer attached to the roof in the upstream region which is moving against the direction of the ventilation. This layer is known as the backlayer. If the ventilation has been increased to the point where the backlayer has disappeared, the stratification is only present downstream of the fire. The velocity at this point is known as the critical ventilation velocity and it represents almost the most controlled situation for a tunnel fire. As the velocity is further increased, the downstream stratification will tend to improve slightly, before degenerating and completely de-stratifying. At this point, the ventilation is causing a greater danger to anything downstream of the fire than if there were no ventilation at all.

Research into the science of tunnel ventilation and smoke control is now considered highly necessary and much study has been put into it ([1] to [12] for example). Develop full scale tests is an extremely costly task and, as with many problems in engineering, modeling procedure permits full scale behavior to be predicted from the information given by small scale tests.

It has previously been well established that the behavior of a tunnel fire can be modeled by using densimetric Froude modeling. In situations in which turbulence is predominant and viscous effects are unimportant (relative indepedence from Reynolds number), only the group $\Delta \rho g \ell / \rho u^{2}$ (Richardson number which is the similarily criterion to be used in all tunnel fire studies) needs to be preserved. This gives the ratio of natural-to-forced convection and as such permits the increased buoyancy forces induced by the flame to be linked to the ventilation velocity via the momentum equation. However, the basic requirement for any scale down experiment is that its results are transferable to the real case it is trying to represent. Specially, one should make sure that the reduction of size does not mask any viscous forces effects.

The relative variation of densities involved in fires are important (for example 0.8 for $T=1200^{\circ} \mathrm{C}$ ). Such values cannot be reached with hydraulic scale models (salt water or 
alcohol models). Aeraulic scale models using helium injection (for example [13]) are more satisfying but the more tempting solution is to simulate the fire by a small burner which power can be varied. In such a way, it is expected that $\Delta \rho / \rho$ is nearly conserved, that turbulent conditions originating from the plume prevail and, therefore, that temperature and velocity fields will be nearly similar for both model an full-scale structures. Assuming that the ideal gas law is applicable, the Richardson number can be written: $R i=\left(T_{\text {ref }}-T_{0}\right) g l / T_{\text {ref }} u^{2}$. It incorporates the velocity $u$ of the longitudinal ventilation, a characteristic reference length $\ell$ of the tunnel and a reference temperature $T_{\text {ref. }}$

The problem remains of how to best define the reference parameters so as to best represent the physical phenomena of the flow. The tunnel height $H$ or hydraulic diameter $D_{H}$ can be selected as the reference length $\ell$. The tunnel height is commonly chosen and the backlayer length $L$ is non-dimensionalized by $L / H$. But the most difficult decision remains in the selection of a suitable reference temperature. It will depend on the effect of heat release and local temperature change due to the heat source. Smoke will move under the influence of buoyancy forces created directly by the fire and under the effects of ventilation. But, if the fire is enough strong for direct flame impingement on the roof, the flame will extend along it flowing above the fresh air. The stratification generated tends to reduce the mixing process and the combustion efficiency. Thus, the "geometry" of the flame with respect to the size of the tunnel and its possible extension under the roof is expected of importance. If, as was expected, there was a temperature whose position and magnitude were linked to both fire wattage and ventilation speed, then it was hoped that using this temperature as $T_{\text {ref }}$ would allow for more meaningful analysis in the subsequent Richardson number calculation.

This study focuses on the selection of this reference temperature and its accuracy in prediction of the flow phenomena. Computational fluid dynamics are also conducted to simulate the flow and give predictions of the smoke backlayer length that can be compared with experimental data from a representation of the (1:30) scale RATP tunnel (metro of Paris).

In the confines of a tunnel, the plume reaches the roof and heat is directed away underneath the cool ceiling, until all heat is transferred away. The work outlines the importance of heat transfer assomptions in the modelling effort. It shows also that the smoke flow dissipates only very slowly as a result of the reduction in buoyancy forces caused by cooling and cutting out of the heat source.

Finally, some additional results are presented verifying the existence of a Critical Ventilation Velocity limit, independent of the power of the fire.

\section{APPARATUS AND INSTRUMENTATION}

\section{The Tunnel}

The experiments are all based around a (1:30) scale model of a typical RATP tunnel, with a slightly simplified geometry to aid manufacturing (Fig. 1).

The 14,2 m tunnel is made up of two separate portions with a wall thickness of $8 \mathrm{~mm}$ :

- one $10 \mathrm{~m}$ section built from Macrolon ${ }^{\mathrm{TM}}$, which, with its temperature limit of $110^{\circ} \mathrm{C}$, is sufficient far from the seat of the fire, 
- another 4,2 m section around the fire source itself, constructed from silica in order to withstand the high levels of temperature reached.

The wall is covered with a foam insulation coat to preserve adiabaticity. The upstream length of the tunnel is such that the flow regime can be assumed to be established and fully turbulent $(\operatorname{Re} \geq 4000)$, with inlet conditions being stabilised via a tranquilisation chamber.

The floor of the tunnel is made from a laminated chipboard so as to minimise conduction of heat from the fire directly to the walls of the tunnel. The mounting plate for the burner itself is a ceramic tile.

\section{The Burner}

The fire is simulated by a porous burner designed to represent as a wide range of fire wattages as possible. The upper part of the burner (75 $\mathrm{mm}$ in diameter) is flushed with the floor. This burner is supplied by butane which flow rate is controlled by a graded ball bearing flowmeter. Due to the turbulence generated by the flame plume, it is expected that for such a small tunnel, laminarization and viscous sublayer phenomena will be unimportant.

\section{The Ventilation System}

A "puller" fan is located downstream of the burner permitting to vary the volumetric air flow rate. A cylindrical section upstream of the tranquilisation chamber allows this flow rate into the tunnel to be measured. This is regulated by a dial connected to the fan motor. The flow rate is calculated by integration of the velocity profile across the tunnel, as measured using Laser Doppler Velocimetry, and this values is correlated to the velocity at the centreline.

\section{Temperature Measurement}

As defined earlier, one aim for these experiments is the possibility of defining $T_{\text {ref }}$ for use in non-dimensional analysis of the problem.

A fine type $\mathrm{K}$ thermocouple $(0,25 \mathrm{~mm}$ in diameter), entering via an axial access slot, allows measurements at particular locations in the median plan of the tunnel, from the floor till the roof. A charly robot is used to achieve its displacement. Run via a PC-based Labview $^{\mathrm{TM}}$ platform enable recording of the average temperature for each point measured. The displacement of the temperature probe is itself controlled by part of the Labview ${ }^{\mathrm{TM}}$ program, as defined by the users resolution and accuracy requirements.

\section{Laser Tomography}

The experimental setup for this technique is also shown is Fig. 1. The laser itself was an argon model which allowed for visualisation of the backlayering length present in the tunnel, the laser sheet entering via a narrow axial access slot through the foam insulation coat. 


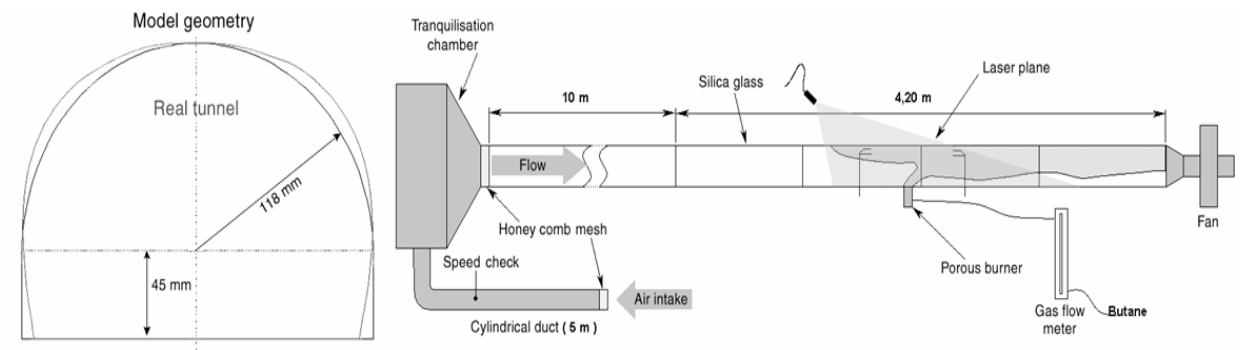

Fig. 1. Tunnel geometry and overall tunnel layout.

\section{NUMERICAL SIMULATION}

The numerical work was achieved using FLUENT 5, 4 software. The simulation of flow around a tunnel fire is particularly complex as it involves numerous coupled physical phenomena. Therefore, the accuracy of the results depends heavily on the careful selection of models: heat source, turbulence, heat transfer to the walls, etc.

The following general assumptions were made: walls were assumed to be adiabatic and smooth, heat transfer by radiation was not modelled, the chemistry of combustion was neglected. A 3-D modelling was used because many of the flow phenomena associated with the backlayer are 3-D in nature or arise from the shape of 3-D objects, e.g., the entrainment of flow around the heat source. In the (1:30) scale RATP tunnel, the fire is produced, as seen above, using a butane burner and this burner spans around 32\% of the tunnel width. The tunnel geometry was created and meshed in GAMBIT. Conditions of symmetry were applied to the median plane of the model which reduces the required computational field by half, and therefore, saves a large amount of computational time. The tunnel inlet was defined as a velocity inlet and simulations were carried out with an established velocity profile obtained from the outlet area of a simulation set up with no fire. In this way, the length of the simulated tunnel can be reduced, and then computational time was saved. The tunnel exit was defined as an outlet boundary and the mass flow rate was the same as the inlet.

In tunnel fire simulation, the heat source (taking not into account combustion) is usually modelled using one of three different heat source models: heated flow element, impinging jet of heated fluid, volume of heat. In this study, the volume method was used because the most realistic. A volume was defined on the floor and a volumetric energy concentration $\left(\mathrm{Wm}^{-3}\right)$ was assigned and thus, by knowing the energy output of the fire, the volumetric energy concentration can be calculated. This method eliminates most problems at the tunnel floor because the energy is already suspended in the path of the flow stream. Since it doesn't involve any momentum input, it will allow the plume to be realistically formed by buoyancy forces alone. But it is not yet known how to select the most appropriate volume of a fire, or if a larger or smaller cube with equal volumetric heat will yield more accurate results. For the purpose of this study, it required only that realistic temperature stratification was achieved. The direct modelling of the fire is not of primary importance. When making direct comparisons to experimental results, Fire Wattage was used instead of volumetric heat release. This was obtained simply by: Volumetric heat $\left(\mathrm{Wm}^{-3}\right)$ x Fire Volume $\left(\mathrm{m}^{3}\right)=$ Fire wattage $(\mathrm{W})$.

The modelling of turbulent flow in the thermally disturbed region (the plume and the backlayer) is one of the most difficult aspects of the numerical simulation. The choice of 
model depends on considerations such as the physics encompassed in the flow, the level of accuracy required, and the time available for the simulation. Two equations models as $k-\varepsilon$ models are those in which the solution of two separate transport equations allows this turbulent velocity and length scales to be independently determined. Among the variants improving the performances of the standard $k-\varepsilon$ model, the RNG $k-\varepsilon$ model, available in FLUENT, uses a rigorous statistical technique. It is shown here as giving the better predictions of backlayer length. In the derivation of the standard $k-\varepsilon$ model, it is assumed that the flow is fully turbulent and the effects of molecular viscosity are negligible. The model is therefore valid only for fully turbulent flows. The RNG $k-\varepsilon$ model is similar in form to the standard model but includes refinement for rapidly strained flows. The main difference between the RNG and standard k- $\varepsilon$ models lies in an additional term in the $\varepsilon$ equation. It is an additional characteristic time enable to introduce a dependence with respect to Reynolds number. Therefore superior performances are expected for the lower Reynolds numbers like those found in the present study. The effects of this term are apparent in regions of high strain rates. In comparaison with the standard $k-\varepsilon$ model, this leads to a higher dissipation rate in the flow and, consequently, a reduction in turbulent kinetic energy $k$. Thus, this model is more responsive to the effects of rapid strain and streamline curvature, which explains its superior performance for the situation of interest.

The following list summarises the conditions used in the simulation: the fire was defined as a volumetric heat source as previously described, the ideal gas equation was used to model the air density, full buoyany effects were enabled, the standard wall functions were used to model near-wall effects.

The FLUENT 5.4 SEGRETATED IMPLICIT solver involving the linearisation of all governing equations (pressure, momentum, energy, turbulence, etc.) was used for each of the individual control volumes. Great attention was devoted into the most efficient selection of discretization methods and under-relaxation factors. Discretization schemas used were mostly first order until the solution had stabilised, whereupon it was possible to change them to second order schemas, which increased the convergence speed (the under-relaxation factors had to be reduced for this to work).

\section{RESULTS}

In order to compare the data from the 3D simulations, the backlayer was chosen as a sensitive representative feature. The standard method for measuring a backlayer length is described below.

Figure 2 shows the $\mathrm{x}$ velocity vectors for a ventilated tunnel fire calculated by the RNG $k-\mathcal{E}$ two equations eddy viscosity model. By definition, the backlayer encompasses any region of flow that propagates from right to left, under ventilation from left to right. The figure shows magnitude of $x$ velocity as length of vector arrow. Defined in terms of $x$ velocity at roof, backlayer length $L$ is the length between the two points at the roof where the velocity is zero. However, it can be seen that the beginning of the backlayer (marked 1 on Fig. 2) is further downstream of the centre of the heat source (marked 2) due to the forced inclination of the heat plume. But the experimental data from the scale model define the backlayer length as the distance from the centre of the heat source to the point upstream where the layer stops. Thus, for comparison purposes, this method will have to be copied and it was assumed that the variation in length of the backlayer downstream of the heat source is negligible. 
Define the upstream tip of the backlayer can be done by plotting the variation of $x$ velocity $u$ at the tunnel roof (see Fig. 3).

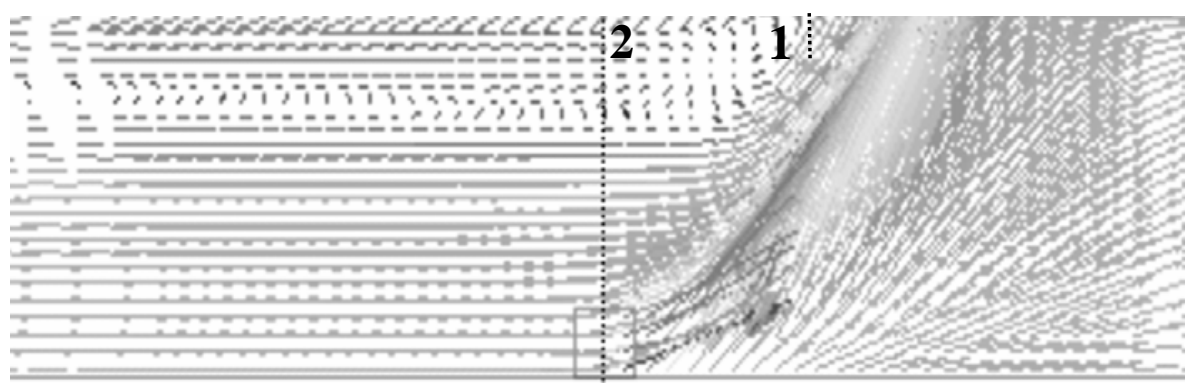

Fig. 2. $x$ - $y$ velocity vectors $\left(P_{f}=600 \mathrm{~W}, u=0,3 \mathrm{~ms}^{-1}\right)$.

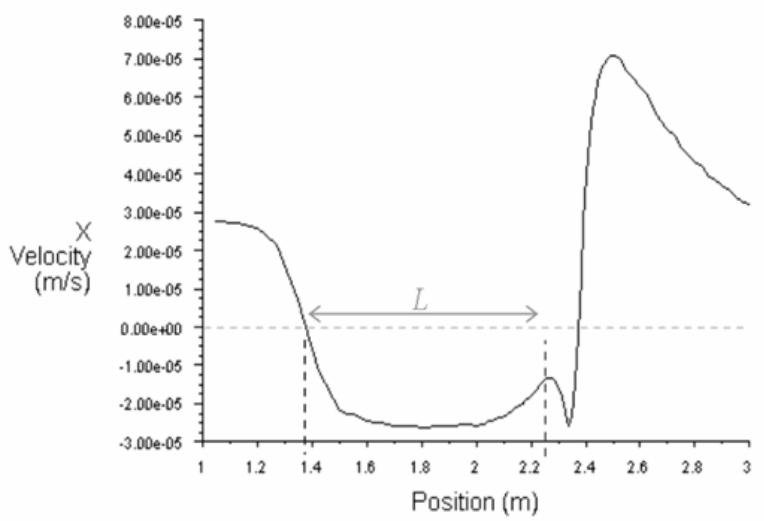

Fig. 3. Plot of $x$-velocity $u$ variation along roof centreline. $L$ represents the backlayer length defined as the distance between its tip $(1,38 \mathrm{~m})$ and the centre of the heat source $(2,25 \mathrm{~m})\left(P_{f}=600 \mathrm{~W}, u=0,3 \mathrm{~ms}^{-1}\right)$.

The length of the backlayer was found to be very sensitive to the magnitude of ventilation velocity, up to the critical velocity where the backlayer disappears. The volumetric heat of the fire was also shown to have a large influence on its size.

It is useful to consider the practicality of obtaining a temperature at a particular location since, in reality, the aim of accurately predicting the backlayer length would rely on an efficient measurement of the reference temperature. For example, in a full-scale tunnel there might be temperature sensors spread out along the roof tunnel; conversely, it is difficult to imagine a practical method of obtaining a temperature at the centre of the fire.

Without eliminating impractical methods at this point, the series of locations to be considered for Reference Temperature are listed below.

Figure 4 provides a visualisation of their relative locations.

-The temperature at the centre of the volumetric heat source, $T_{\text {source }}$ (1). This has little practical application although it should characterise the fire reasonably well and may yield links between fire and backlayer. 
-The temperature at the roof directly above the centre of the heat source, $T_{\text {roof }}$

(2). This is more practical although it would involve the precise location of the fire.

-The maximum temperature at any point of the tunnel roof centerline $T_{\text {roof } \max }$ (3). It is the most practically sound location.

-The temperature at the point of the roof where the backlayer begins, i.e., where velocity is zero, $T_{\text {stag }}$ (4). The selection of a stagnation reference point may prove to be useful although it would be difficult to locate in reality.

-The mean temperature of the plume, $T_{b u l k}$, calculated from the energy equation as follows: $T_{b u l k}-T_{0}=P f / \rho A u C p$ with $P_{f}$ power of the fire, $A$ tunnel section, $u$ longitudinal ventilation velocity, $\rho$ density, $C p$ specific heat and $T_{0}$ ambient temperature.

The values of all fire temperatures were recorded for each of the simulations carried out. The backlayer length $L$, non-dimensionalized using the tunnel height $H$, was calculated for each simulation and plotted against the five different definitions of Richardson number. A line of best fit was calculated for each version of Richardson number and the correlation between the line and the data points were measured using the square of the Pearsons Product Moment Coefficient.

Figure 5 displays how backlayer length relates to Richardson number and the correlative strength of each $T_{\text {ref. }}$.

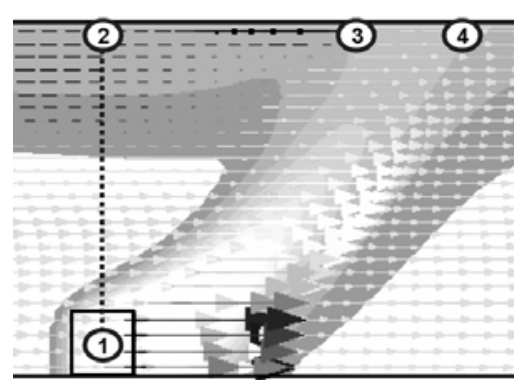

Fig. 4. Location of Reference

Temperatures, superimposed over greyscale temperature contours and $x$ velocity vectors. Number refer to definitions above.

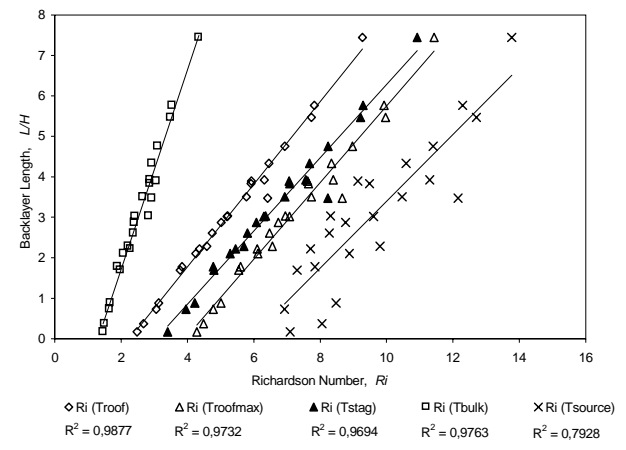

Fig. 5. Graph of Richardson number vs. backlayer length calculated using the five different reference temperatures. R2 correlation values are shown.

It can be seen that using $T_{\text {source }}$ to calculate $R i$ is of little use, whilst selection of $T_{\text {roof }}$, $T_{\text {roofmax }}, T_{\text {stag }}$ or $T_{\text {bulk }}$ gives a strong correlation. It should be noted that while $T_{\text {bulk }}$ is a mean value calculated directly from the heat release rate of the source, the rest of the points represent discrete locations around the tunnel and therefore incorporate different physical influences. In a practical situation, obtaining the value of $T_{\text {bulk }}$ would be difficult, whilst the selection of $T_{\text {roofmax }}, T_{\text {roof }}$ and $T_{\text {stag }}$ is much more feasible (temperature sensors along the roof centreline).

The results from the two correlations found using $T_{\text {bulk }}$ and $T_{\text {roof }}$ are compared to experimental data from the (1:30) scale experimental tunnel (Fig. 6). All the experimental data used are from scenarios that used a similar range of heat release and ventilation velocity as were used in the numerical simulations. It is seen a satisfying correlation 
between experimental and numerical results for $R i$ calculated using $T_{\text {bulk }}$ whilst the other definition of reference temperature, $T_{\text {roof }}$, is shown to provide results out of agreement. This is clearly due, by using $T_{b u l k}$, to the direct calculation of the temperature change from the power of the heat source.

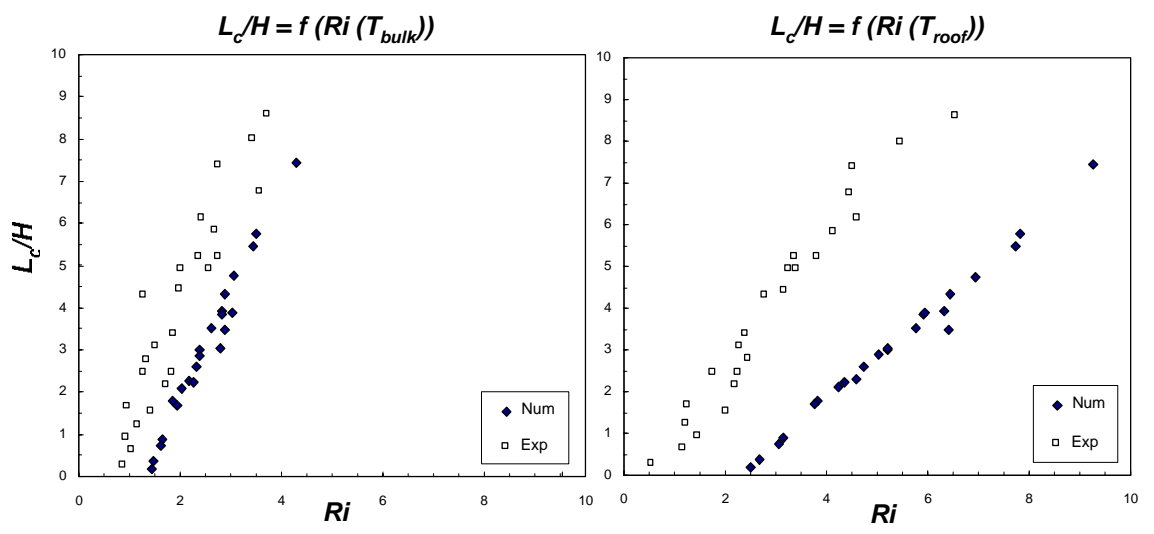

Fig. 6. Comparison of Richardson number calculated from both experimental and computational data using reference temperatures Tbulk and Troof.

The use of a temperature obtained directly from the heat source provides logically the most accurate result. In contrast, high uncertainties can be associated with results obtained through measurements of temperature at discrete locations along the roof, problem that is inherent in the experimental technique (nature and location of the probe). Accurately predicting the backlayer length would rely on an efficient measurement of the reference temperature. Nevertheless, even with the use of $T_{b u k}$, the prediction of the backlayer length appears slightly underestimated. This is likely due to the fact that backlayer propagates as gravity current along the tunnel roof, the strength of which depending on the rate of heat generation but also strongly on the heat losses to the tunnel walls and roof. The experimental observation shows that backlayer length is very sensitive to these losses and, hence, the numerical simulation is probably affected by inaccurate heat transfer assumption. Taking into account the radiation effects might, for instance, render the model more performant, especially in the downstream region. Close to the fire, heat transfer analysis can only be ascertained by carrying out thermal radiation in addition to convection. But the role of the former tends to vanish as the temperature of the smoke decreases. In the future, much more attention must be paid to well account for heat transfers in the modelling efforts.

This main role of heat transfer is well evidenced considering thermal inertia of the backlayer. Experiments conducted in conditions where a backlayer is formed show that a sudden extinction of the fire (by cutting off butane supply) is followed by the persistance of smoke flow under the roof during periods of time that can be long. Figure 7 shows for example the evolution of $T_{\text {roof }}$ and $T_{M}$ at a given position $M x / H=1,2$, and $y / H=0,9$ within the backlayer, for a fire power of $1000 \mathrm{~W}$ and a ventilation velocity of $0,36 \mathrm{~ms}^{-1}$, after the extinction of the burner. Three steps are observed. First, the temperatures $T_{\text {roof }}$ and $T_{M}$ stay practically stable that is significant of the backlayering persistence. Next, temperature vanishes abruptly due to the smoke layer dissipation for lack of supply. The third step is a quasi linear decrease of the two temperatures till ambient, $T_{M}$ characterising 
then the thermal boundary layer induced by the roof. During this last phase, the ventilation current cools flow and tunnel walls by forced convection.

The results from such tests reinforce the statement of the strong dependence of backlayering (length, spreading, stratification) on the heat transfer characteristics of the tunnel. In real situation, this must be well apprehended when determining safety control strategies.

Current practice, as aforementioned, is commonly increasing the ventilation velocity to the point where the backlayer has disappeared and all heat flows downstream of the fire. The velocity at this point is known as the Critical Ventilation Velocity $u_{c}$. It represents almost the most controlled situation for a fire tunnel. In reality, the risk is to see the downstream roof layer become unattached (de-stratification). In addition, the extra ventilation may also providing the fire with a lot-more oxygen and therefore the fire is growing.

The Critical Ventilation Velocity was determined for different powers of the fire. In fact, it appeared difficult to precisely set the velocity at which backflow disappears. When the backlayer length is shortened under about $20 \mathrm{~cm}$, it becomes difficult to stabilize it. It is very unstable, fluctuating randomly in length in such a way that the measurements are only time averaged with an uncertainty associated with each value up to about $20 \%$. Nevertheless, an acceptable order of magnitude estimate is obtained. Figure 8 shows the evolution of Critical Ventilation Velocity determined in this way as a function of the heat release rate. One significant feature is that the evolution shows an asymptotical trend, suggesting the existence of a Critical Ventilation Velocity limit. The correlation of the data gives the following expression : $u_{c}=0,57\left(1-\exp \left(-0,037 P_{f}\right)\right.$, where $u_{c}$ is expressed in $\mathrm{ms}^{-1}$ and $P$ in $W$.

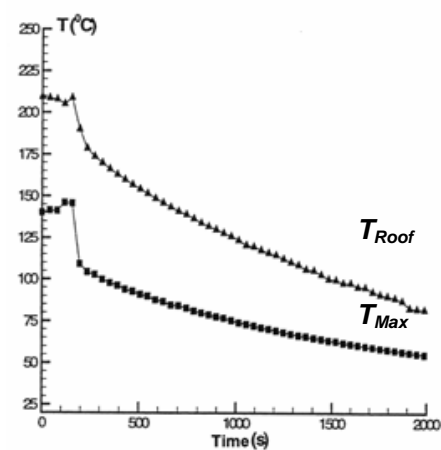

Fig. 7. Supporting evidence of thermal inertia of backlayer.

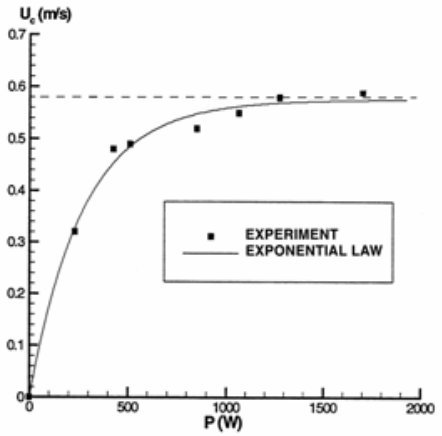

Fig. 8. Limiting critical ventilation velocity.

The deduced limiting Critical Ventilation Velocity, called "Super Critical," is 0,57 ms'. A survey of previous published data shows that this value compares favorably with values obtained from other scale models: $0,45 \mathrm{~ms}^{-1}$ by Oka and Atkinson [14] and $0,47 \mathrm{~ms}^{-1}$ by Mégret [13]. These two limiting rates are yet a little bite lower. This is mainly due to the use of "cold" scale models by these authors.

Danziger and Kennedy [15] were the first to conceptualize the notion of "Super Critical Ventilation Velocity." Assuming that the density of hot gases tends towards zero for fires of very large sizes, they defined a Critical Richardson number $R i_{c}$ which does not depend on the heat release rate $R i_{c} \rightarrow g H / u_{s c}^{2}$ (inverse of Froude number). 
From our results $R i_{c}$ becomes 4, 9, value that lies in the range 4, 5-6, 7 proposed by Lee et al. [16].

Finally, on the basis of the scaling law which can be used to compare behavior in scale model and real situation (for similarity $u$ must be divided by 0,18 ), a Super Critical Ventilation Velocity of $3,2 \mathrm{~ms}^{-1}$ is deduced. This value is in the order of magnitude estimate commonly recommended in determining fire safety strategies to push all the smoke produced downstream of a fire.

\section{CONCLUSION}

The accuracy of the simulations has been shown to be confident in term of prediction of backlayer length. The quantitative prediction of the flow stratification is good whilst the qualitative detail is not always as accurate. The gravitational damping of turbulence in the backlayer is found to be larger than expected, and the RNG $k-\varepsilon$ model is found to give better predictions than the standard $k-\varepsilon$ model, because although it might not be simulating the exact flow, its under-prediction of turbulence is in this case more accurate.

The following conclusions can be drawn from the work completed in this study:

- the fire can be considered to be nothing more than a source of heat and this one can be modelled effectively by using the volumetric heat source approximation,

- the use of $T_{b u l k}$, obtained directly from the heat source, provides the most accurate definition of reference temperature for the correlation between backlayer length and Richardson number, although its practicallity of is limited,

- the results obtained through the numerical simulation of the computational tunnel are shown to give some reasonably good levels of prediction for the experimental results obtained from the scale tunnel.

However, even if the FLUENT RNG $k-\varepsilon$ model is clearly preferable for the present buoyancy controlled plume, it subsists the doubt on how this model predictions for a $0.163 \mathrm{~m}$ high tunnel compare with predictions for a full-scale tunnel when strictly following Froude modeling principles. Specially, do the backlayer length reported for the experiment correspond to those of a full-scale situation? This is still an unsolved and controversial problem and further research on this question is required.

To simplify the study, the effects of radiation and combustion have been ignored. However, further investigations would require for a more rigorous model to be used, which will account for these effects. The tunnel walls in the (1:30) scale experimental model are covered with a foam insulation coat to insure adiabaticity and preserve experimental conditions. In fact the tunnel model does not mimic accurately this heat transfer situation and this aspect needs also to be improved.

In other respects, the existence of a Super Critical Ventilation Velocity for which there is no flow back against the air current, is verified. In real situation it corresponds to a value of $u \geq 3,2 \mathrm{~ms}^{-1}$. But it must be highlighted that ventilating much above this velocity may lead to destratification of the flow, which is undesirable.

\section{REFERENCES}

[1] Thomas, P.H., "The Movement of Smoke in Horizontal Passages against an Air Flow,” Fire Research Note, N23, Fire research Station, Watford, UK, 1968. 
[2] Heselden, A.J.M., "Studies of Fire and Smoke Behavior Relevant to Tunnels. Paper J1," $2^{\text {nd }}$ Int. Symp. on Aerodynamics and Ventilation of Vehicle Tunnels, Cambridge, U.K., 1976.

[3] Hwang, C.C., and Wargo, J.D., "Experimental Study of Thermally Generated Reverse Stratified Layers in a Fire Tunnel," Combustion and Flame, 1986, 66, pp. 171-180.

[4] Guelzim, A., Souil, J.M., Vantelon, J.P., Doan Kim Son, Gabay, D., and Dallest, D., "Modelling of a Reverse Layer of Fire-induced Smoke in a Tunnel," Fire Safety Science-Proceedings of the Fourth International Symposium, 1994, pp. 277-288.

[5] Fletcher, D.F., Kent, J.H., Apte, V.B., and Green, A.R., "Numerical Simulations of Smoke Movement from a Pool Fire in a Ventilated Tunnel," Fire Safety Journal, 1994, 23, pp. 305-325.

[6] Charters, D.A., Gray, W.A., and McIntosh, A.C., "A Computer Model to Assess Fire Hazards in Tunnels (FASIT),” Fire Technology, 1994, 30, pp. 134-154.

[7] Bettis, R.J., Daish, N., Jagger, S.F., and Lindon, P.F., "Control of Smoke Movement Close to a Tunnel Fire, Safety in Road and Rail Tunnels,” Second International Conference, Granada, Spain, 1995, Proceedings, pp. 311-319.

[8] Woodburn, P.J., and Britter, R.E., "CFD Simulations of a Tunnel Fire. Part I," Fire Safety Journal, 1996, 26, pp. 35-62. Part II, Fire Safety Journal, 1996, 26, pp. 63-90.

[9] Wu, Y., and Bakar, M.Z.A., "Control of Smoke Flow in Tunnel Fires Using Longitudinal Ventilation Systems. A study of the Critical Velocity,” Fire Safety Journal, 2000, 35, pp. 363-390.

[10] Xue, H., Chew, T.C., Tay, K.L., and Cheng, Y.M., "Control of Ventilation Airflow for Tunnel Fire Safety,” Combustion Science and Technology, 2000, 152, pp. 179-196.

[11] Schabacker, J., Bettelini, M., and Rudin, Ch., "CFD Study of Temperature and Smoke Distribution in a Railway Tunnel with Natural Ventilation System," Tunnel Management International Journal, 2002, Vol. 5, Issue 3.

[12] Li, J.S.M., and Chow, W.K., "Numerical Studies on Performance Evaluation of Tunnel Ventilation Safety Systems," Tunnelling and Underground Space Technology, 2003, 18, pp. 435-452.

[13] Megret, O., "Etude expérimentale de la propagation des fumées d'incendie en tunnel pour différents systèmes de ventilation,” $\mathrm{PhD}$ Thesis, University of Valencienne, France, October 20, 1999.

[14] Oka, Y., and Atkinson, G.T., "Control of Smoke Flow in Tunnel Fires," Fire Safety Journal, 1995, 25, pp. 305-322.

[15] Danziger, N.H., and Kennedy, W.D., "Longitudinal Ventilation Analysis for the Glenwood Canyon Tunnel," Proceedings of the $4^{\text {th }}$ Int. Symp. Aerodynamics \& Ventilation of Vehicle Tunnels, 1982, pp. 169-186.

[16] Lee, C.K., Hwang, C.C., Singer, J.M., and Chaiken, R.F., The Second International Mine Ventilation Congress, 1979, pp. 448. 